\title{
Some Properties of Solutions of Second-Order Linear Differential Equations
}

\author{
Zinelaâbidine Latreuch and Benharrat Belaïdi \\ Laboratory of Pure and Applied Mathematics, Department of Mathematics, \\ University of Mostaganem (UMAB), BP 227, 27000 Mostaganem, Algeria \\ Correspondence should be addressed to Benharrat Belaïdi; belaidi@univ-mosta.dz
}

Received 13 August 2012; Accepted 25 September 2012

Academic Editor: Rabha W. Ibrahim

Copyright (C) 2013 Z. Latreuch and B. Belaïdi. This is an open access article distributed under the Creative Commons Attribution License, which permits unrestricted use, distribution, and reproduction in any medium, provided the original work is properly cited.

We study the growth and oscillation of $g_{f}=d_{1} f_{1}+d_{2} f_{2}$, where $d_{1}$ and $d_{2}$ are entire functions of finite order not all vanishing identically and $f_{1}$ and $f_{2}$ are two linearly independent solutions of the linear differential equation $f^{\prime \prime}+A(z) f=0$.

\section{Introduction and Main Results}

Throughout this paper, we assume that the reader is familiar with the fundamental results and the standard notations of the Nevanlinna value distribution theory (see [1-4]). In addition, we will use $\lambda(f)$ and $\bar{\lambda}(f)$ to denote, respectively, the exponents of convergence of the zero sequence and distinct zeros of a meromorphic function $f, \rho(f)$ to denote the order of growth of $f$.

Definition 1 (see $[4,5])$. Let $f$ be a meromorphic function. Then the hyperorder of $f(z)$ is defined by

$$
\rho_{2}(f)=\limsup _{r \rightarrow+\infty} \frac{\log \log T(r, f)}{\log r} .
$$

Definition 2 (see $[4,5])$. Let $f$ be a meromorphic function. Then the hyper-exponent of convergence of zeros sequence of $f(z)$ is defined by

$$
\lambda_{2}(f)=\limsup _{r \rightarrow+\infty} \frac{\log \log N(r, 1 / f)}{\log r},
$$

where $N(r, 1 / f)$ is the counting function of zeros of $f(z)$ in $\{z:|z|<r\}$. Similarly, the hyperexponent of convergence of the sequence of distinct zeros of $f(z)$ is defined by

$$
\bar{\lambda}_{2}(f)=\limsup _{r \rightarrow+\infty} \frac{\log \log \bar{N}(r, 1 / f)}{\log r},
$$

where $\bar{N}(r, 1 / f)$ is the counting function of distinct zeros of $f(z)$ in $\{z:|z|<r\}$.

Suppose that $f_{1}$ and $f_{2}$ are two linearly independent solutions of the complex linear differential equation

$$
f^{\prime \prime}+A(z) f=0,
$$

and the polynomial of solutions

$$
g_{f}=d_{1} f_{1}+d_{2} f_{2},
$$

where $A$ and $d_{j}(j=1,2)$ are entire functions of finite order in the complex plane. It is clear that if $d_{j}(j=1,2)$ are complex numbers or $d_{1}=c d_{2}$ where $c$ is a complex number, then $g_{f}$ is a solution of (4) or has the same properties of the solutions.

It is natural to ask what can be said about the properties of $g_{f}$ in the case when $d_{1} \neq c d_{2}$ where $c$ is a complex number and under what conditions $g_{f}$ keeps the same properties of the solutions of (4).

In [6], Chen studied the fixed points and hyper-order of solutions of second-order linear differential equations with entire coefficients and obtained the following results.

Theorem A (see [6]). For all nontrivial solutions $f$ of (4) the following hold. 
(i) If $A$ is a polynomial with $\operatorname{deg} A=n \geq 1$, then one has

$$
\lambda(f-z)=\rho(f)=\frac{n+2}{2} .
$$

(ii) If $A$ is transcendental and $\rho(A)<\infty$, then one has

$$
\begin{gathered}
\lambda(f-z)=\rho(f)=\infty, \\
\lambda_{2}(f-z)=\rho_{2}(f)=\rho(A) .
\end{gathered}
$$

Before we state our results we define $h$ and $\psi$ by

$$
\begin{gathered}
h=\left|\begin{array}{cccc}
d_{1} & 0 & d_{2} & 0 \\
d_{1}^{\prime} & d_{1} & d_{2} & d_{2,} \\
d_{1}^{\prime \prime}-d_{1} A & 2 d_{1}^{\prime} & d_{2}^{\prime \prime}-d_{2} A & 2 d_{2}^{\prime} \\
d_{1}^{\prime \prime}-3 d_{1}^{\prime} A-d_{1} A^{\prime} & d_{1}^{\prime \prime}-d_{1} A+2 d_{1}^{\prime \prime} & d_{2}^{\prime \prime \prime}-3 d_{2}^{\prime} A-d_{2} A^{\prime} & d_{2}^{\prime \prime}-d_{2} A+2 d_{2}^{\prime \prime}
\end{array}\right|, \\
\psi(z)=\frac{2\left(d_{1} d_{2} d_{2}^{\prime}-d_{2}^{2} d_{1}^{\prime}\right)}{h} \varphi^{(3)}+\phi_{2} \varphi^{\prime \prime}+\phi_{1} \varphi^{\prime}+\phi_{0} \varphi,
\end{gathered}
$$

where $\varphi \equiv 0$ is entire function of finite order and

$$
\begin{gathered}
\phi_{2}=\frac{3 d_{2}^{2} d_{1}^{\prime \prime}-3 d_{1} d_{2} d_{2}^{\prime \prime}}{h}, \\
\phi_{1}=\frac{2 d_{1} d_{2} d_{2}^{\prime} A+6 d_{2} d_{1}^{\prime} d_{2}^{\prime \prime}-6 d_{2} d_{2}^{\prime} d_{1}^{\prime \prime}-2 d_{2}^{2} d_{1}^{\prime} A}{h}, \\
\phi_{0}=\frac{2 d_{2} d_{1}^{\prime} d_{2}^{\prime \prime \prime}-2 d_{1} d_{2}^{\prime} d_{2}^{\prime \prime \prime}-3 d_{1} d_{2} d_{2}^{\prime \prime} A-3 d_{2} d_{1}^{\prime \prime} d_{2}^{\prime \prime}}{h} \\
+\frac{2 d_{1} d_{2} d_{2}^{\prime} A^{\prime}-4 d_{2} d_{1}^{\prime} d_{2}^{\prime} A-6 d_{1}^{\prime} d_{2}^{\prime} d_{2}^{\prime \prime}+3 d_{1}\left(d_{2}^{\prime \prime}\right)^{2}}{h} \\
+\frac{4 d_{1}\left(d_{2}^{\prime}\right)^{2} A+3 d_{2}^{2} d_{1}^{\prime \prime} A+6\left(d_{2}^{\prime}\right)^{2} d_{1}^{\prime \prime}-2 d_{2}^{2} d_{1}^{\prime} A^{\prime}}{h} .
\end{gathered}
$$

The subject of this paper is to study the controllability of solutions of the differential equation (4). In fact, we study the growth and oscillation of $g_{f}=d_{1} f_{1}+d_{2} f_{2}$ where $f_{1}$ and $f_{2}$ are two linearly independent solutions of (4) and $d_{1}$ and $d_{2}$ are entire functions of finite order not all vanishing identically and satisfying $d_{1} \neq c d_{2}$ where $c$ is a complex number, and we obtain the following results.

Theorem 3. Let $A(z)$ be a transcendental entire function of finite order. Let $d_{j}(z)(j=1,2)$ be finite-order entire functions that are not all vanishing identically such that $\max \left\{\rho\left(d_{1}\right), \rho\left(d_{2}\right)\right\}<\rho(A)$. If $f_{1}$ and $f_{2}$ are two linearly independent solutions of (4), then the polynomial of solutions (5) satisfies

$$
\begin{gathered}
\rho\left(g_{f}\right)=\rho\left(f_{j}\right)=\infty \quad(j=1,2), \\
\rho_{2}\left(g_{f}\right)=\rho_{2}\left(f_{j}\right)=\rho(A) \quad(j=1,2) .
\end{gathered}
$$

Theorem 4. Under the hypotheses of Theorem 3, let $\varphi(z) \equiv 0$ be an entire function with finite order such that $\psi(z) \equiv 0$. If $f_{1}$ and $f_{2}$ are two linearly independent solutions of (4), then the polynomial of solutions (5) satisfies

$$
\begin{gathered}
\bar{\lambda}\left(g_{f}-\varphi\right)=\lambda\left(g_{f}-\varphi\right)=\rho\left(f_{j}\right)=\infty \quad(j=1,2), \\
\bar{\lambda}_{2}\left(g_{f}-\varphi\right)=\lambda_{2}\left(g_{f}-\varphi\right)=\rho_{2}\left(f_{j}\right)=\rho(A) \quad(j=1,2) .
\end{gathered}
$$

Theorem 5. Let $A(z)$ be a polynomial of $\operatorname{deg} A=n$. Let $d_{j}(z)(j=1,2)$ be finite-order entire functions that are not all vanishing identically such that $h \equiv 0$ and $\max \left\{\rho\left(d_{1}\right), \rho\left(d_{2}\right)\right\}<$ $(n+2) / 2$. If $f_{1} f_{2}$ are two linearly independent solutions of $(4)$, then the polynomial of solutions (5) satisfies

$$
\rho\left(g_{f}\right)=\rho\left(f_{j}\right)=\frac{n+2}{2} \quad(j=1,2) .
$$

Theorem 6. Under the hypotheses of Theorem 5, let $\varphi(z) \equiv 0$ be an entire function with $\rho(\varphi)<(n+2) / 2$ such that $\psi(z) \equiv 0$. If $f_{1}$ and $f_{2}$ are two linearly independent solutions of (4), then the polynomial of solutions (5) satisfies

$$
\bar{\lambda}\left(g_{f}-\varphi\right)=\lambda\left(g_{f}-\varphi\right)=\frac{n+2}{2} .
$$

\section{Auxiliary Lemmas}

Lemma 7 (see $[7,8]$ ). Let $A_{0}, A_{1}, \ldots, A_{k-1}, F \equiv 0$ be finiteorder meromorphic functions. If $f$ is a meromorphic solution of the equation

$$
f^{(k)}+A_{k-1} f^{(k-1)}+\cdots+A_{1} f^{\prime}+A_{0} f=F
$$

with $\rho(f)=+\infty$ and $\rho_{2}(f)=\rho$, then $f$ satisfies

$$
\begin{aligned}
& \bar{\lambda}(f)=\lambda(f)=\rho(f)=+\infty, \\
& \bar{\lambda}_{2}(f)=\lambda_{2}(f)=\rho_{2}(f)=\rho .
\end{aligned}
$$
in [9].

Here, we give a special case of the result due to Cao et al.

Lemma 8. Let $A_{0}, A_{1}, \ldots, A_{k-1}, F \equiv 0$ be finite-order meromorphic functions. If $f$ is a meromorphic solution of (14) with

$$
\max \left\{\rho\left(A_{j}\right)(j=0,1, \ldots, k-1), \rho(F)\right\}<\rho(f)<+\infty,
$$

then

$$
\bar{\lambda}(f)=\lambda(f)=\rho(f) .
$$

\section{Proofs of the Theorems}

Proof of Theorem 3. Suppose that $f_{1}$ and $f_{2}$ are two linearly independent solutions of (4). Then by Theorem A, we have

$$
\begin{gathered}
\rho\left(f_{1}\right)=\rho\left(f_{2}\right)=\infty, \\
\rho_{2}\left(f_{1}\right)=\rho_{2}\left(f_{2}\right)=\rho(A) .
\end{gathered}
$$


Suppose that $d_{1}=c d_{2}$, where $c$ is a complex number. Then, by (5) we obtain

$$
g_{f}=c d_{2} f_{1}+d_{2} f_{2}=\left(c f_{1}+f_{2}\right) d_{2} .
$$

Since $f=c f_{1}+f_{2}$ is a solution of $(4)$ and $\rho\left(d_{2}\right)<\rho(A)$, then we have

$$
\begin{gathered}
\rho\left(g_{f}\right)=\rho\left(c f_{1}+f_{2}\right)=\infty, \\
\rho_{2}\left(g_{f}\right)=\rho_{2}\left(c f_{1}+f_{2}\right)=\rho(A) .
\end{gathered}
$$

Suppose now that $d_{1} \neq c d_{2}$ where $c$ is a complex number. Differentiating both sides of (5), we obtain

$$
g_{f}^{\prime}=d_{1}^{\prime} f_{1}+d_{1} f_{1}^{\prime}+d_{2}^{\prime} f_{2}+d_{2} f_{2}^{\prime}
$$

Differentiating both sides of (21), we obtain

$$
g_{f}^{\prime \prime}=d_{1}^{\prime \prime} f_{1}+2 d_{1}^{\prime} f_{1}^{\prime}+d_{1} f_{1}^{\prime \prime}+d_{2}^{\prime \prime} f_{2}+2 d_{2}^{\prime} f_{2}^{\prime}+d_{2} f_{2}^{\prime \prime} \text {. }
$$

Substituting $f_{j}^{\prime \prime}=-A f_{j}(j=1,2)$ into (22), we obtain

$$
g_{f}^{\prime \prime}=\left(d_{1}^{\prime \prime}-d_{1} A\right) f_{1}+2 d_{1}^{\prime} f_{1}^{\prime}+\left(d_{2}^{\prime \prime}-d_{2} A\right) f_{2}+2 d_{2}^{\prime} f_{2}^{\prime} \text {. }
$$

Differentiating both sides of (23) and by substituting $f_{j}^{\prime \prime}=$ $-A f_{j}(j=1,2)$, we obtain

$$
\begin{aligned}
g_{f}^{\prime \prime \prime}= & \left(d_{1}^{\prime \prime \prime}-3 d_{1}^{\prime} A-d_{1} A^{\prime}\right) f_{1}+\left(d_{1}^{\prime \prime}-d_{1} A+2 d_{1}^{\prime \prime}\right) f_{1}^{\prime} \\
& +\left(d_{2}^{\prime \prime \prime}-3 d_{2}^{\prime} A-d_{2} A^{\prime}\right) f_{2}+\left(d_{2}^{\prime \prime}-d_{2} A+2 d_{2}^{\prime \prime}\right) f_{2}^{\prime}
\end{aligned}
$$

By (5), (21), (23), and (24) we have

$$
\begin{gathered}
g_{f}=d_{1} f_{1}+d_{2} f_{2}, \\
g_{f}^{\prime}=d_{1}^{\prime} f_{1}+d_{1} f_{1}^{\prime}+d_{2}^{\prime} f_{2}+d_{2} f_{2}^{\prime}, \\
g_{f}^{\prime \prime}=\left(d_{1}^{\prime \prime}-d_{1} A\right) f_{1}+2 d_{1}^{\prime} f_{1}^{\prime}+\left(d_{2}^{\prime \prime}-d_{2} A\right) f_{2}+2 d_{2}^{\prime} f_{2}^{\prime}, \\
g_{f}^{\prime \prime \prime}=\left(d_{1}^{\prime \prime \prime}-3 d_{1}^{\prime} A-d_{1} A^{\prime}\right) f_{1}+\left(d_{1}^{\prime \prime}-d_{1} A+2 d_{1}^{\prime \prime}\right) f_{1}^{\prime} \\
+\left(d_{2}^{\prime \prime \prime}-3 d_{2}^{\prime} A-d_{2} A^{\prime}\right) f_{2}+\left(d_{2}^{\prime \prime}-d_{2} A+2 d_{2}^{\prime \prime}\right) f_{2}^{\prime} .
\end{gathered}
$$

To solve this system of equations, we need first to prove that $h \equiv 0$. By simple calculations we obtain

$$
\begin{aligned}
h= & \left|\begin{array}{cccc}
d_{1} & 0 & d_{2} & 0 \\
d_{1}^{\prime} & d_{1} & d_{2}^{\prime} & d_{2} \\
d_{1}^{\prime \prime}-d_{1} A & 2 d_{1}^{\prime} & d_{2}^{\prime \prime}-d_{2} A & 2 d_{2}^{\prime} \\
d_{1}^{\prime \prime \prime}-3 d_{1}^{\prime} A-d_{1} A^{\prime} & d_{1}^{\prime \prime}-d_{1} A+2 d_{1}^{\prime \prime} & d_{2}^{\prime \prime \prime}-3 d_{2}^{\prime} A-d_{2} A^{\prime} & d_{2}^{\prime \prime}-d_{2} A+2 d_{2}^{\prime \prime}
\end{array}\right| \\
= & \left(4 d_{1}^{2}\left(d_{2}^{\prime}\right)^{2}+4 d_{2}^{2}\left(d_{1}^{\prime}\right)^{2}-8 d_{1} d_{2} d_{1}^{\prime} d_{2}^{\prime}\right) A+2 d_{1} d_{2} d_{1}^{\prime} d_{2}^{\prime \prime \prime} \\
& +2 d_{1} d_{2} d_{2}^{\prime} d_{1}^{\prime \prime \prime}-6 d_{1} d_{2} d_{1}^{\prime \prime} d_{2}^{\prime \prime}-6 d_{1} d_{1}^{\prime} d_{2}^{\prime} d_{2}^{\prime \prime}-6 d_{2} d_{1}^{\prime} d_{2}^{\prime} d_{1}^{\prime \prime} \\
& +6 d_{1}\left(d_{2}^{\prime}\right)^{2} d_{1}^{\prime \prime}+6 d_{2}\left(d_{1}^{\prime}\right)^{2} d_{2}^{\prime \prime}-2 d_{2}^{2} d_{1}^{\prime} d_{1}^{\prime \prime \prime}-2 d_{1}^{2} d_{2}^{\prime} d_{2}^{\prime \prime \prime} \\
& +3 d_{1}^{2}\left(d_{2}^{\prime \prime}\right)^{2}+3 d_{2}^{2}\left(d_{1}^{\prime \prime}\right)^{2} .
\end{aligned}
$$

To show that $4 d_{1}^{2}\left(d_{2}^{\prime}\right)^{2}+4 d_{2}^{2}\left(d_{1}^{\prime}\right)^{2}-8 d_{1} d_{2} d_{1}^{\prime} d_{2}^{\prime} \equiv 0$, we suppose that

$$
d_{1}^{2}\left(d_{2}^{\prime}\right)^{2}+d_{2}^{2}\left(d_{1}^{\prime}\right)^{2}-2 d_{1} d_{2} d_{1}^{\prime} d_{2}^{\prime}=0
$$

Dividing both sides of (27) by $\left(d_{1} d_{2}\right)^{2}$, we obtain

$$
\left(\frac{d_{2}^{\prime}}{d_{2}}\right)^{2}+\left(\frac{d_{1}^{\prime}}{d_{1}}\right)^{2}-2 \frac{d_{1}^{\prime}}{d_{1}} \frac{d_{2}^{\prime}}{d_{2}}=0
$$

equivalent to

$$
\left(\frac{d_{1}^{\prime}}{d_{1}}-\frac{d_{2}^{\prime}}{d_{2}}\right)^{2}=0,
$$

which implies that $d_{1}=c d_{2}$ where $c$ is a complex number and this is a contradiction. Since $\max \left\{\rho\left(d_{1}\right), \rho\left(d_{2}\right)\right\}<\rho(A)$ and $4 d_{1}^{2}\left(d_{2}^{\prime}\right)^{2}+4 d_{2}^{2}\left(d_{1}^{\prime}\right)^{2}-8 d_{1} d_{2} d_{1}^{\prime} d_{2}^{\prime} \equiv 0$, we can deduce from (26) that

$$
\rho(h)=\rho(A)>0 .
$$

Hence $h \equiv 0$. By Cramer's method we have

$$
\begin{aligned}
f_{1} & =\frac{\left|\begin{array}{cccc}
g_{f} & 0 & d_{2} & 0 \\
g_{f}^{\prime} & d_{1} & d_{2}^{\prime} & d_{2} \\
g_{f}^{\prime \prime} & 2 d_{1}^{\prime} & d_{2}^{\prime \prime}-d_{2} A & 2 d_{2}^{\prime} \\
g_{f}^{\prime \prime} & d_{1}^{\prime \prime}-d_{1} A+2 d_{1}^{\prime \prime} & d_{2}^{\prime \prime \prime}-3 d_{2}^{\prime} A-d_{2} A^{\prime} & d_{2}^{\prime \prime}-d_{2} A+2 d_{2}^{\prime \prime}
\end{array}\right|}{h} \\
& =\frac{2\left(d_{1} d_{2} d_{2}^{\prime}-d_{2}^{2} d_{1}^{\prime}\right)}{h} g_{f}^{(3)}+\phi_{2} g_{f}^{\prime \prime}+\phi_{1} g_{f}^{\prime}+\phi_{0} g_{f},
\end{aligned}
$$

where $\phi_{j}(j=0,1,2)$ are meromorphic functions of finite order defined in (9). Suppose now that $\rho\left(g_{f}\right)<\infty$, then by (31) we obtain $\rho\left(f_{1}\right)<\infty$, which is a contradiction, hence $\rho\left(g_{f}\right)=\infty$. By (5) we have $\rho_{2}\left(g_{f}\right) \leq \rho(A)$. Suppose that $\rho_{2}\left(g_{f}\right)<\rho(A)$, then by (31) we obtain $\rho_{2}\left(f_{1}\right)<\rho(A)$, which is a contradiction. Hence $\rho_{2}\left(g_{f}\right)=\rho(A)$. 
Proof of Theorem 4. By Theorem 3 we have $\rho\left(g_{f}\right)=\infty$ and $\rho_{2}\left(g_{f}\right)=\rho(A)$. Set $w(z)=d_{1} f_{1}+d_{2} f_{2}-\varphi$. Since $\rho(\varphi)<\infty$, then we have $\rho(w)=\rho\left(g_{f}\right)=\infty$ and $\rho_{2}(w)=\rho_{2}\left(g_{f}\right)=\rho(A)$. In order to prove that $\bar{\lambda}\left(g_{f}-\varphi\right)=\lambda\left(g_{f}-\varphi\right)=\infty, \bar{\lambda}_{2}\left(g_{f}-\varphi\right)=$ $\lambda_{2}\left(g_{f}-\varphi\right)=\rho(A)$ we need to prove only that $\bar{\lambda}(w)=\lambda(w)=$ $\infty$ and $\bar{\lambda}_{2}(w)=\lambda_{2}(w)=\rho(A)$. By $g_{f}=w+\varphi$ we get from (31)

$$
f_{1}=\frac{2\left(d_{1} d_{2} d_{2}^{\prime}-d_{2}^{2} d_{1}^{\prime}\right)}{h} w^{(3)}+\phi_{2} w^{\prime \prime}+\phi_{1} w^{\prime}+\phi_{0} w+\psi,
$$

where

$$
\psi=\frac{2\left(d_{1} d_{2} d_{2}^{\prime}-d_{2}^{2} d_{1}^{\prime}\right)}{h} \varphi^{(3)}+\phi_{2} \varphi^{\prime \prime}+\phi_{1} \varphi^{\prime}+\phi_{0} \varphi .
$$

Substituting (32) into (4), we obtain

$$
\frac{2\left(d_{1} d_{2} d_{2}^{\prime}-d_{2}^{2} d_{1}^{\prime}\right)}{h} w^{(5)}+\sum_{j=0}^{4} \beta_{j} w^{(j)}=-\left(\psi^{\prime \prime}+A \psi\right)=B
$$

where $\beta_{j}(j=0, \ldots, 4)$ are meromorphic functions of finite order. Since $\psi \neq 0$ and $\rho(\psi)<\infty$, it follows that $\psi$ is not a solution of (4), which implies that $B \equiv 0$. Then by applying Lemma 7 we obtain (11).

Proof of Theorem 5. Suppose that $f_{1}$ and $f_{2}$ are two linearly independent solutions of (4). Then by Theorem A

$$
\rho\left(f_{1}\right)=\rho\left(f_{2}\right)=\frac{n+2}{2} \text {. }
$$

By the same reasoning as in Theorem 3, we have

$$
h=\left|\begin{array}{cccc}
d_{1} & 0 & d_{2} & 0 \\
d_{1}^{\prime} & d_{1} & d_{2} & d_{2} \\
d_{1}^{\prime \prime}-d_{1} A & 2 d_{1}^{\prime} & d_{2}^{\prime \prime}-d_{2} A & 2 d_{2}^{\prime} \\
d_{1}^{\prime \prime \prime}-3 d_{1}^{\prime} A-d_{1} A^{\prime} & d_{1}^{\prime \prime}-d_{1} A+2 d_{1}^{\prime \prime} & d_{2}^{\prime \prime \prime}-3 d_{2}^{\prime} A-d_{2} A^{\prime} & d_{2}^{\prime \prime}-d_{2} A+2 d_{2}^{\prime \prime}
\end{array}\right| .
$$

Since $h \neq 0$ and by Cramer's method we have

$$
\begin{aligned}
f_{1} & =\frac{\left|\begin{array}{cccc}
g_{f} & 0 & d_{2} & 0 \\
g_{f}^{\prime} & d_{1} & d_{2}^{\prime} & d_{2} \\
g_{f}^{\prime \prime} & 2 d_{1}^{\prime} & d_{2}^{\prime \prime}-d_{2} A & 2 d_{2}^{\prime} \\
g_{f}^{\prime \prime} & d_{1}^{\prime \prime}-d_{1} A+2 d_{1}^{\prime \prime} & d_{2}^{\prime \prime \prime}-3 d_{2}^{\prime} A-d_{2} A^{\prime} & d_{2}^{\prime \prime}-d_{2} A+2 d_{2}^{\prime \prime}
\end{array}\right|}{h} \\
& =\frac{2\left(d_{1} d_{2} d_{2}^{\prime}-d_{2}^{2} d_{1}^{\prime}\right)}{h} g_{f}^{(3)}+\phi_{2} g_{f}^{\prime \prime}+\phi_{1} g_{f}^{\prime}+\phi_{0} g_{f},
\end{aligned}
$$

where $\phi_{j}(j=0,1,2)$ are meromorphic functions with $\rho\left(\phi_{j}\right)<(n+2) / 2(j=0,1,2)$ defined in (9). By (5) we have $\rho\left(g_{f}\right) \leq(n+2) / 2$. Suppose that $\rho\left(g_{f}\right)<(n+2) / 2$, then by (37) we obtain $\rho\left(f_{1}\right)<(n+2) / 2$, which is a contradiction. Hence, $\rho\left(g_{f}\right)=(n+2) / 2$.
Proof of Theorem 6. By Theorem 5 we have $\rho\left(g_{f}\right)=(n+2) / 2$. Set $w(z)=d_{1} f_{1}+d_{2} f_{2}-\varphi$. Since $\rho(\varphi)<(n+2) / 2$, then we have $\rho(w)=\rho\left(g_{f}\right)=(n+2) / 2$. In order to prove that $\bar{\lambda}\left(g_{f}-\varphi\right)=(n+2) / 2$, we need to prove only that $\bar{\lambda}(w)=$ $(n+2) / 2$. By $g_{f}=w+\varphi$ we get from (37)

$$
\begin{aligned}
f_{1}= & \frac{2\left(d_{1} d_{2} d_{2}^{\prime}-d_{2}^{2} d_{1}^{\prime}\right)}{h} w^{(3)} \\
& +\phi_{2} w^{\prime \prime}+\phi_{1} w^{\prime}+\phi_{0} w+\psi,
\end{aligned}
$$

where

$$
\begin{aligned}
\psi= & \frac{2\left(d_{1} d_{2} d_{2}^{\prime}-d_{2}^{2} d_{1}^{\prime}\right)}{h} \varphi^{(3)} \\
& +\phi_{2} \varphi^{\prime \prime}+\phi_{1} \varphi^{\prime}+\phi_{0} \varphi
\end{aligned}
$$

Substituting (38) into (4), we obtain

$$
\frac{2\left(d_{1} d_{2} d_{2}^{\prime}-d_{2}^{2} d_{1}^{\prime}\right)}{h} w^{(5)}+\sum_{j=0}^{4} \beta_{j} w^{(j)}=-\left(\psi^{\prime \prime}+A \psi\right)=B,
$$

where $\beta_{j}(j=0, \ldots, 4)$ are meromorphic functions with $\rho\left(\beta_{j}\right)<(n+2) / 2$. Since $\psi \neq 0$ and $\rho(\psi)<(n+2) / 2$, it follows that $\psi$ is not a solution of (4), which implies that $B \equiv 0$. Then by applying Lemma 8 we obtain (13).

\section{Acknowledgments}

The authors would like to thank the referees for their helpful remarks and suggestions to improve the paper. This research is supported by Agence Nationale pour le Développement de la Recherche Universitaire (ANDRU) and University of Mostaganem (UMAB), (PNR Project Code 8/u27/3144).

\section{References}

[1] W. K. Hayman, Meromorphic Functions, Clarendon Press, Oxford, UK, 1964.

[2] I. Laine, Nevanlinna Theory and Complex Differential Equations, vol. 15 of de Gruyter Studies in Mathematics, Walter de Gruyter, Berlin, Germany, 1993.

[3] R. Nevanlinna, Eindeutige analytische Funktionen, vol. 46 of Die Grundlehren der mathematischen Wissenschaften, Springer, Berlin, Germany, 2nd edition, 1974.

[4] C. C. Yang and H. X. Yi, Uniqueness Theory of Meromorphic Functions, vol. 557 of Mathematics and its Applications, Kluwer Academic Publishers, Dordrecht, The Netherlands, 2003.

[5] Z. X. Chen and C. C. Yang, "Some further results on the zeros and growths of entire solutions of second order linear differential equations," Kodai Mathematical Journal, vol. 22, no. 2, pp. 273-285, 1999.

[6] Z. X. Chen, "The fixed points and hyper-order of solutions of second order complex differential equations," Acta Mathematica Scientia A, vol. 20, no. 3, pp. 425-432, 2000 (Chinese). 
[7] B. Belaïdi, "Growth and oscillation theory of solutions of some linear differential equations," Matematicki Vesnik, vol. 60, no. 4, pp. 233-246, 2008.

[8] Z. X. Chen, "Zeros of meromorphic solutions of higher order linear differential equations," Analysis, vol. 14, no. 4, pp. 425-438, 1994.

[9] T. B. Cao, Z. X. Chen, X. M. Zheng, and J. Tu, "On the iterated order of meromorphic solutions of higher order linear differential equations," Annals of Differential Equations, vol. 21, no. 2, pp. 111-122, 2005. 


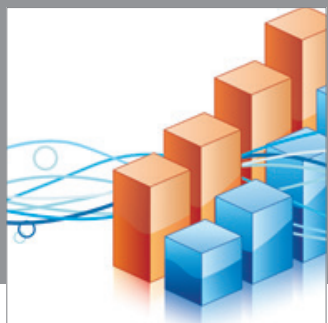

Advances in

Operations Research

mansans

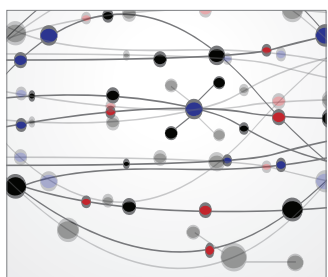

The Scientific World Journal
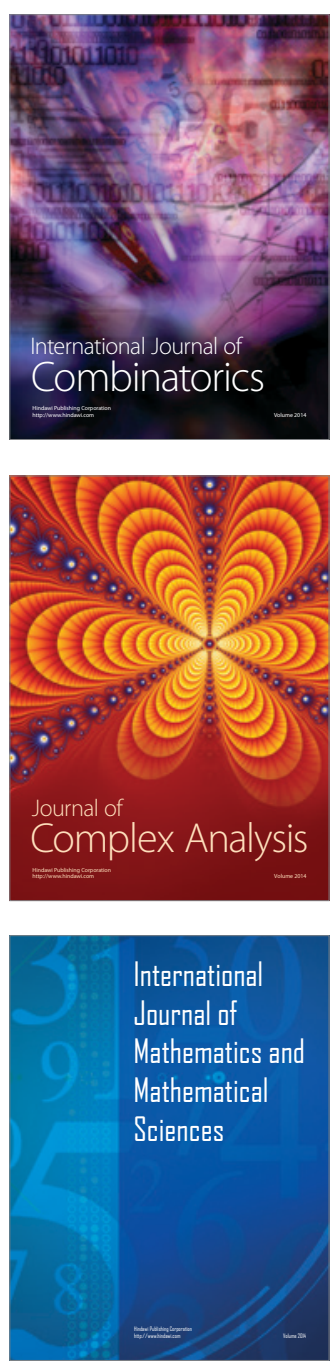
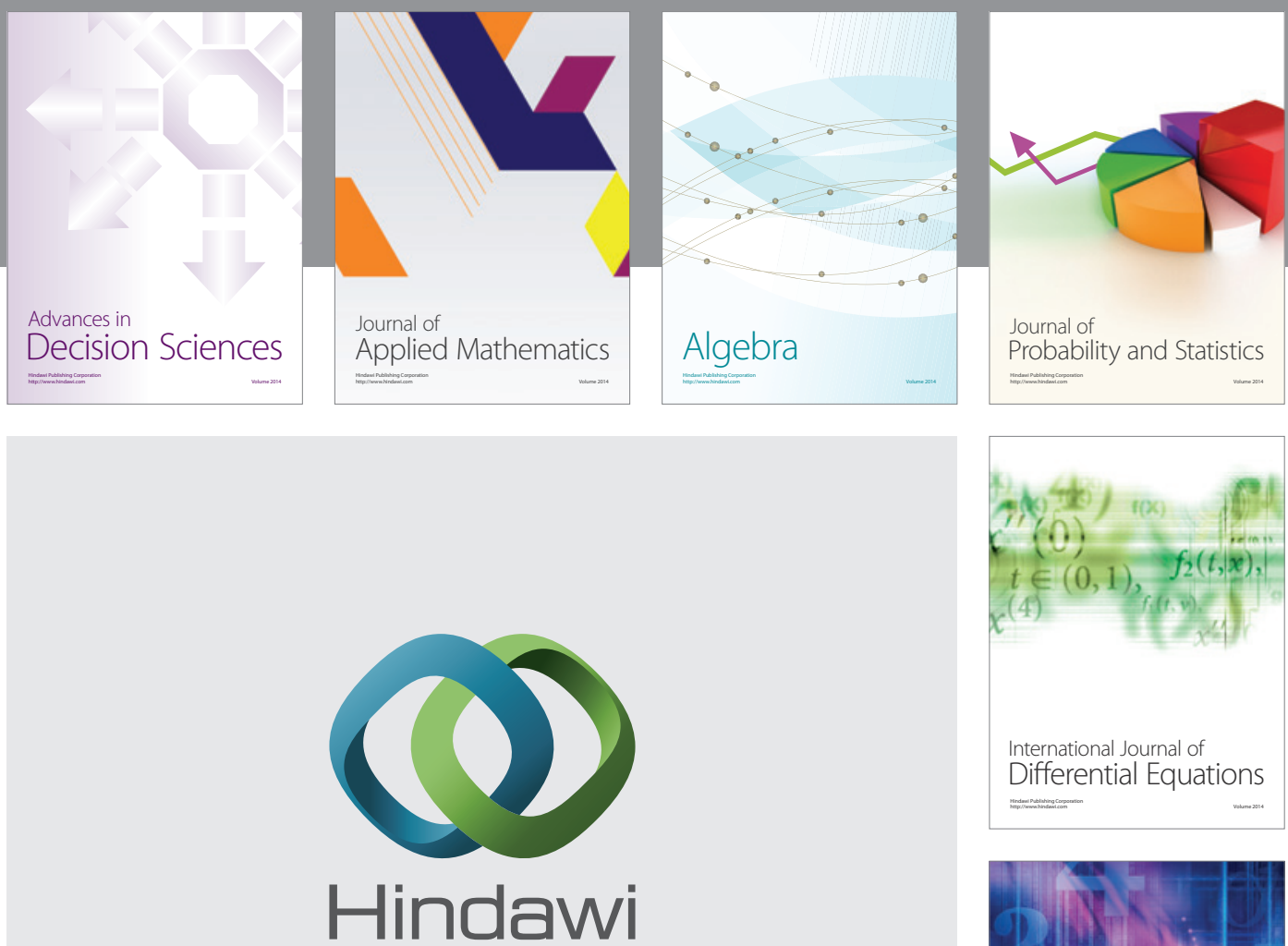

Submit your manuscripts at http://www.hindawi.com
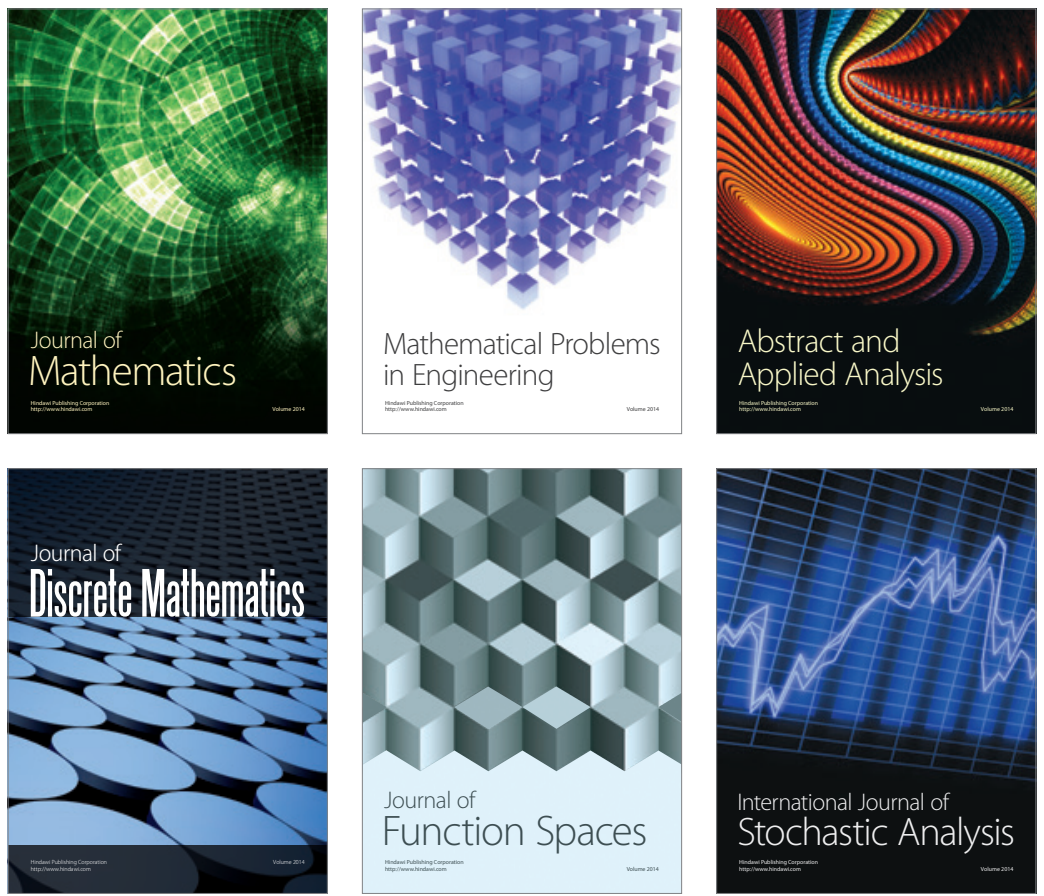

Journal of

Function Spaces

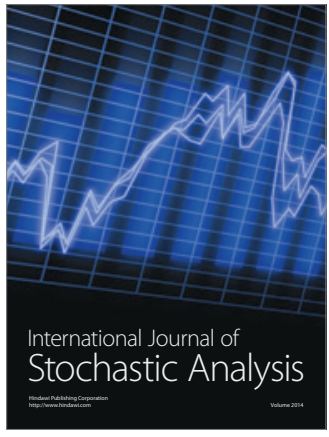

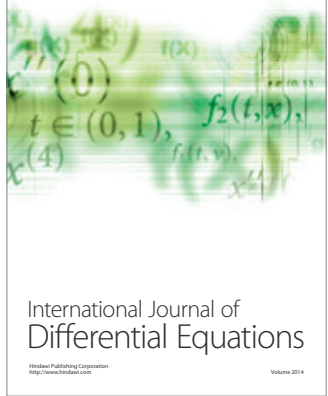
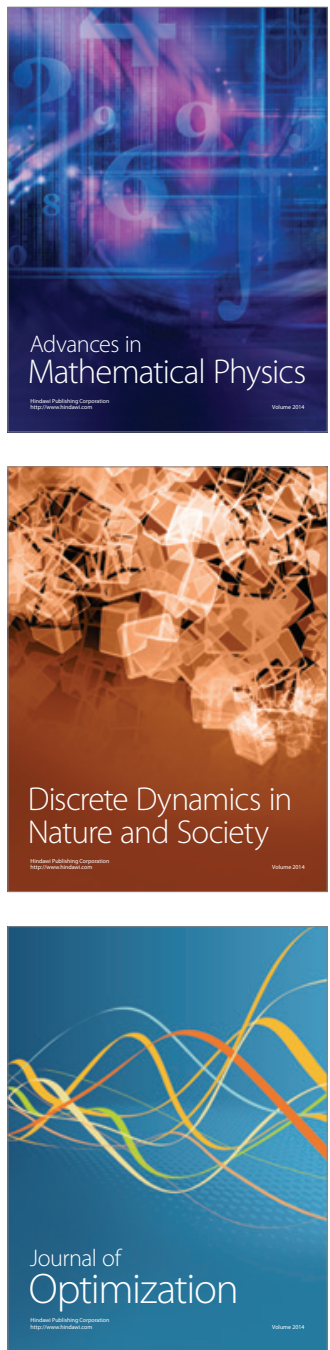